\title{
The prognostic value of seminal anti-sperm antibodies screening in men prepared for ICSI: a call to change the current antibody-directed viewpoint of sperm autoimmunity testing
}

\author{
Ahmed F. El-Sherbiny (D), Tamer A. Ali iD, Eman A. Hassan, Amira B. Mehaney \\ and Heba A. Elshemy
}

\begin{abstract}
Aims: We aimed to prospectively evaluate the prognostic value of seminal anti-sperm antibodies (ASA) screening in couples prepared for intracytoplasmic sperm injection (ICSI). Methods: A prospective comparative study was conducted, including 184 non-azoospermic infertile men scheduled for ICSI. For each patient, detection of ASA in seminal plasma using the ELISA technique was done and its relation to standard semen parameters and ICSI outcomes, including fertilization, embryo development, and pregnancy rates, was analyzed. Results: Sperm count, motility, and morphology were negatively affected by existence of seminal ASA ( $p=0.012,0.006$, and 0.011 , respectively). However, no statistically significant difference was detected between patients with positive and negative seminal ASA regarding the median values of fertilization ( $56.3 \%$ versus $66.7 \%, p=0.091)$, percentage of couples with grade A embryo development $(91.4 \%$ versus $89.9 \%, p=0.520)$, and pregnancy rates $(31.4 \%$ versus $32.2 \%, p=0.98$ ) after ICSI.

Conclusion: ICSI seems able to overcome the problem of ASA in semen. The routine screening of ASA in men prepared for ICSI has no additional prognostic value and cannot be recommended for the time being, until more specific antigen-concerned testing can be developed.
\end{abstract}

Keywords: anti-sperm antibodies, autoimmunity, immunologic infertility, semen parameters, sperm

Received: 19 July 2020; revised manuscript accepted: 26 November 2020.
Tamer A. Al
Al-Azhar University, Cairo,
Egypt
dr_tamer_alidayahoo.com
Ahmed F. El-Sherbiny Department of Andrology,
International Islamic
Center for Population
Studies and Research,
Al-Azhar University, Cairo,
Egypt
Eman A. Hassan Amira B. Mehaney Department of Embryology, International Islamic Center for Population Studies and Research, Al-Azhar University, Cairo, Egypt Heba A. Elshemy Department of Dermatology, Venereology and Andrology, Faculty of Medicine, Al-Azhar University, Cairo, Egypt

\section{Introduction}

Since their recognition in 1899 by the Nobel Prize laureates Metchnikoff and Landsteiner, more than 100 sperm surface auto- and isoantigens have been identified. ${ }^{1}$ Most of these antigens are specific to the sperm, while, surprisingly, some of them can be found in other tissues such as ovaries, brain, placenta, and malignant tumors. ${ }^{2}$ Due to their delayed establishment, a long time beyond completion of the process of self-tolerance, sperm surface antigens are recognized by immune cells

as foreign antigens that may trigger immune reaction if the anatomical and immunological protective barriers were not functioning properly, leading to secretion of anti-sperm antibodies (ASA) by activated B-lymphocytes. ${ }^{3}$ It was demonstrated that ASA can impede fertility at different levels, ${ }^{4}$ as they may affect sperm motility, capacitation, or acrosome reaction ${ }^{3}$ or inhibit sperm-oocyte interaction by attacking antigens involved in these functions, ${ }^{5}$ or they may act indirectly by facilitating the release of inflammatory mediators that 
impair the fertilizing ability of sperm. ${ }^{6}$ Interestingly, the negative impact of ASA was reported to also affect post-fertilization events such as embryo development and implantation after conventional in-vitro fertilization (IVF) ${ }^{5,7}$ It was noted that this negative effect was dose-dependent. ${ }^{8}$ Contrarily, significant ASA levels have been also identified in some fertile men, ${ }^{9}$ indicating that not all ASA subtypes impair sperm functions, depending on their antigenic specificity, which is, unfortunately, not testable by available methods. ${ }^{10}$ Before the era of gamete micromanipulation techniques, several studies advocated that ASA may be associated with a reduced probability of pregnancy with intrauterine insemination (IUI), despite using different semen processing techniques to elute ASA attached to the sperm surface, namely, dilution, cryopreservation, and antigenic competition, ${ }^{11}$ which were not sufficient to reduce ASA load, due to their high affinity to the corresponding antigens, leading to great difficulty in their separation without harming the sperm. ${ }^{12}$ The studies that have evaluated the relationship between ASA and IVF outcomes reported contradictory results, ranging from no effect to significant negative effect, ${ }^{13,14}$ which may be explained by the fact that some, but not all, antibodies may interfere with sperm-zona interaction or oolemma merging needed for successful fertilization in conventional IVF. ${ }^{15}$ Following the introduction of intracytoplasmic sperm injection (ICSI) to the field of assisted reproduction, it became the preferred alternative for IVF in the management of couples suffering from immunological infertility, supported by the general belief that, unlike IUI and IVF, ICSI could bypass all steps likely to be affected by autoimmunity. Based on this belief, the question considering the impact of ASA on ICSI outcomes was not investigated extensively. ${ }^{16}$ Currently, there is no consensus among clinicians on the practical significance of ASA screening on ICSI outcomes, considering that infertile couples often resort to ICSI for indications other than immunological infertility. ${ }^{15}$ Bearing in mind the previously reported negative implications of autoimmunity on post-fertilization events in IVF, ${ }^{5,7}$ we sought to prospectively evaluate the prognostic value of ASA screening in ICSI cycles, by comparing ICSI outcomes between groups with positive and negative autoantibodies in semen.

\section{Material and methods}

This prospective study included 184 couples, between November 2017 and October 2019. All subjects were attendants of the Assisted Reproductive Technology (ART) unit of the International Islamic Center for Population Studies and Research (IICPSR), Al-Azhar University, Cairo, Egypt. The study was approved by the Institutional Ethics Committee, Department of Andrology, International Islamic Center for Population Studies and Research, (affiliated to Al-Azhar University, Egypt) (REC number: 2862). Written informed consent was obtained from all participants and the collected data were managed and analyzed confidentially and anonymously.

After initial gynecological assessment, couples with female partners $>35$ years or with any apparent causes of infertility that may negatively affect the results, or addressed as potentially poor responder [menstrual day 3 follicle-stimulating hormone $(\mathrm{FSH})>10 \mathrm{IU} / \mathrm{ml}$, antral follicle count $<6$ or anti-Mullerian hormone $<1.5 \mathrm{ng} / \mathrm{ml}$ ] were excluded from the study. Semen samples, obtained on the day of ova pick up, were subdivided into two unequal portions; the largest one was used for ICSI, while the smallest part was utilized for conventional semen analysis and detection of ASA in seminal plasma. Semen analyses were performed in all patients according to World Health Organization (WHO) 2010 guidelines. ${ }^{17}$

\section{Sample collection and storage}

Semen samples after conventional examination were centrifuged for $10 \mathrm{~min}$ at $1000 \mathrm{rpm}$ within $30 \mathrm{~min}$ of collection, and supernatants were collected and kept in refrigerator at $\leqslant-20^{\circ} \mathrm{C}$ for collective measurement of the whole samples by the enzyme-linked immunosorbent assay (ELISA) method later.

\section{Detection of ASA in seminal plasma by ELISA technique}

The ELISA plate is coated with a mix of spermatozoa proteins which are recognized by antispermatozoa antibodies. Standards and samples are pipetted into the wells and then incubated in a humidified chamber to prevent liquid loss due to evaporation. During this incubation, antispermatozoa antibodies bind to the spermatozoa proteins and are thus immobilized on the plate. After washing away any unbound substances, the enzyme conjugate, a Horse Radish Peroxidase- (HRP-) conjugated human antibody 
is added to each well and incubated. After washing away any unbound antibody-enzyme reagent, a substrate solution, Tetramethylbenzidine (TMB), is added to the wells. Solution color changes from blue to yellow in proportion to the amount of antibodies bound in the initial step. The intensity of the color is measured using BioTek ${ }^{\circledR}$ microplate reader at a wavelength of $450 \pm 2 \mathrm{~nm}$. The results were calculated automatically using a 4 PL (4 Parameter Logistics) curve compatible with internal software of BioTek $^{\circledR}$ microplate reader, considering normal values as $0-60 \mathrm{U} / \mathrm{ml}$ and elevated (positive) values if $>60 \mathrm{U} / \mathrm{ml}$.

\section{Intracytoplasmic sperm injection}

Ovulation induction was carried out using a combination of gonadotropin-releasing hormone agonist and human menopausal gonadotropin. Ovulation was triggered by human chorionic gonadotropin (HCG), when $\geqslant 3$ follicles measured $\geqslant 18 \mathrm{~mm}$ in diameter and serum estradiol concentration was at least $1000 \mathrm{ng} / \mathrm{L}$. Oocyte retrieval was carried out $36 \mathrm{~h}$ later, followed by oocyte assessment for maturity under an inverted microscope, then preparation for ICSI, which include removal of the cumulus and corona radiata cells (denudation). Every mature oocyte was injected with a single, living, immobilized spermatozoon, then fertilization was confirmed $16-18 \mathrm{~h}$ later by observation of two distinct pronuclei $(2 \mathrm{PN})$ and two polar bodies. The fertilization rate was calculated as the number of fertilized oocytes divided by the total number of mature oocytes for each couple. Fertilized oocytes were observed for embryonic development on day 3 after injection, just prior to the transfer, and classified as previously described ${ }^{18}$ into:

- Good quality grade A embryos (0-20\% of the volume filled with anucleated fragments).

- Fair quality grade B embryos (20-50\% anucleated fragments).

- Poor quality grade $\mathrm{C}$ embryos ( $>50 \%$ anucleated fragments).

The best available embryos of the first two grades were eligible for transfer. Serum HCG level was measured 14 days later to distinguish a positive biochemical pregnancy. In such cases, clinical pregnancy was reaffirmed sonographically 5-6 weeks post-injection.

\section{Statistical methods}

Data were analyzed using IBM $^{\odot}$ SPSS $^{\odot}$ Statistics version 23 (IBM ${ }^{\circ}$ Corp., Armonk, NY, USA). Normally distributed numerical data were presented as mean and SD and intergroup differences were compared using the unpaired $t$-test. Non-normally distributed numerical data were presented as median and interquartile range (IQR), and intergroup differences were compared using the Wilcoxon rank sum test (for 2-group comparison) or the Jonckheere-Terpstra trend test (for multiple-group comparison). Categorical data were presented as number and percentage and differences were compared using Fisher's exact test (for nominal data) or the chisquared test (for ordinal data). Correlations were tested using the Spearman rank correlation. Receiver operating characteristic (ROC) curve analysis was used to examine the predictive value of seminal ASA.

\section{Results}

The prevalence of seminal ASA among participants was $19 \%(35 / 184)$. There was a highly significant difference between patients of both groups of negative and positive ASA regarding the median values of sperm count $(\mathrm{mill} / \mathrm{ml}) 25$ (IQR 12-34) versus 10 (IQR 5-20), ( $p=0.012)$, total motility 38 (IQR 20-50) versus 20 (IQR 10$30),(p=0.006)$, and morphology 90 (IQR 8597) versus 98 (IQR 95-100), $(p=0.011)$, as the three parameters were negatively affected by the existence of seminal ASA. However, seminal volume and pus cells were not affected by the existence of ASA ( $p=0.294$ and 0.111 , respectively) (Table 1). Although the median value of fertilization rate was lower in patients with positive ASA compared with those with negative ASA, representing $56.3 \%$ and $66.7 \%$, respectively, the difference did not reach statistical significance $(p=0.091)$ (Table 1). There was no statistically significant difference between groups regarding the median number of embryos of different grades (A, B, and C, $p=0.717,0.712$, and 0.712 , respectively) or the grade of best embryo produced among the studied population $(p=0.340)$ (Table 1). Among 35 positive cases of seminal ASA, 11 patients $(31.4 \%)$ achieved clinical pregnancy, while the other 24 patients $(68.6 \%)$ failed to get pregnant. On other hand, among 149 patients negative for seminal ASA, 48 cases $(32.2 \%)$ achieved clinical pregnancy, while the other 101 patients $(67.8 \%)$ failed to get pregnant. There was no statistically significant difference 
Table 1. Relation between seminal ASA and semen parameters, fertilization rate, embryo development and pregnancy rate.

\begin{tabular}{|c|c|c|c|c|c|}
\hline \multirow[t]{2}{*}{ Variable } & \multicolumn{2}{|c|}{ Positive seminal ASA ( $n=35$ ) } & \multicolumn{2}{|c|}{ Negative seminal ASA $(n=149)$} & \multirow[t]{2}{*}{$p$-value* } \\
\hline & Median & IQR & Median & IQR & \\
\hline Volume (ml) & $(1.0-2.5)$ & 2.0 & $(1.5-2.5)$ & 2.0 & 0.294 \\
\hline Sperm count (mill/ml) & $(5-20)$ & 10 & $(12-34)$ & 25 & 0.012 \\
\hline Motility (\%) & $(10-30)$ & 20 & $(20-50)$ & 38 & 0.006 \\
\hline Abnormal forms (\%) & $(95-100)$ & 98 & (85-97) & 90 & 0.011 \\
\hline Pus cells (cells/HPF) & $(5-9)$ & 7 & $(3-7)$ & 5 & 0.111 \\
\hline Seminal ASA (U/ml) & $(81-128)$ & 113 & $(18-34)$ & 26 & $<0.001$ \\
\hline Fertilization rate $(\%)$ & $(40-66.7)$ & 56.3 & $(50-100)$ & 66.7 & 0.091 \\
\hline No. of Grade A embryos & $(1-3)$ & 2 & $(1-3)$ & 2 & 0.717 \\
\hline No. of Grade B embryos & $(0-1)$ & 1 & $(0-1)$ & 1 & 0.712 \\
\hline No. of Grade C embryos & $(0-1)$ & 1 & $(0-1)$ & 1 & 0.712 \\
\hline $\begin{array}{l}\text { Couples best embryo } \\
\text { grade } A\end{array}$ & \multicolumn{2}{|l|}{$32(91.4 \%)$} & \multicolumn{2}{|l|}{$134(89.9 \%)$} & 0.520 \\
\hline $\begin{array}{l}\text { Couples best embryo } \\
\text { grade B }\end{array}$ & \multicolumn{2}{|l|}{$2(5.7 \%)$} & \multicolumn{2}{|l|}{$11(7.4 \%)$} & \\
\hline $\begin{array}{l}\text { Couples best embryo } \\
\text { grade C }\end{array}$ & \multicolumn{2}{|l|}{$1(2.9 \%)$} & \multicolumn{2}{|l|}{$4(2.7 \%)$} & \\
\hline Negative pregnancy & \multicolumn{2}{|l|}{$24(68.6 \%)$} & \multicolumn{2}{|l|}{$101(67.8 \%)$} & $0.98 * *$ \\
\hline Positive pregnancy & \multicolumn{2}{|l|}{$11(31.4 \%)$} & \multicolumn{2}{|l|}{$48(32.2 \%)$} & \\
\hline \multicolumn{5}{|c|}{$\begin{array}{l}\text { Data are median and interquartile range (IQR) or number }(\%) . \\
\text { *Wilcoxon rank sum test. } \\
\text { **Fisher's exact test. } \\
\text { ASA, anti-sperm antibodies; HPF, high-power field }\end{array}$} & \\
\hline
\end{tabular}

between groups regarding pregnancy rates $(p=0.98)$ (Table 1). No significant difference was detected between couples with distinct best-produced embryos ( $\mathrm{A}, \mathrm{B}$, or $\mathrm{C}$ ) regarding the median values of seminal ASA levels [27 (IQR 18-43) versus 28 (IQR 18-41) versus 22 (IQR 18-28) U/ml, respectively, $p=0.663$ ] (Table 2 ). Also, there was no significant difference between patients with negative and positive clinical pregnancy $(n=125$ and 59 , respectively) as regards the median values of seminal ASA levels 26 (IQR 18-37) and 28 (IQR 14-44) U/ml, respectively, $(p=0.556)$ (Table 2). Seminal ASA level had poor predictive value of clinical pregnancy with an area under the ROC curve (AUC) of 0.529 ( $p$-value $=0.559)$ (Figure 1).

\section{Discussion}

Despite the great advancement in the field of assisted reproduction in recent years, there are still many unknown and uncharacterized factors that unquestionably play a significant role in the pathogenesis of unexplained infertility and repeated ICSI failure. One of the suspected factors is the existence of ASA. ${ }^{19}$ However, there is uncertainty about this possible causal relationship due to the observation of detectable ASA in a significant proportion of fertile men. Moreover, some reports argued a possible beneficial effect of some ASA on the fertilization process, through activation of specific surface key antigens and through promotion of adhesion and penetration of sperm to oocyte. ${ }^{20}$ Review of the previous studies 
Table 2. Relation between seminal ASA level and the best embryo quality produced and positive or negative pregnancy.

\begin{tabular}{|c|c|c|c|c|}
\hline \multirow[t]{2}{*}{ Variable } & \multicolumn{3}{|c|}{ Couples best embryo } & \multirow[t]{2}{*}{$p$-value } \\
\hline & Grade $A(n=166)$ & Grade B $(n=13)$ & Grade C $(n=5)$ & \\
\hline \multirow[t]{3}{*}{ ASA level (U/ml) } & $27(18-43)$ & $28(18-41)$ & $22(18-28)$ & $0.663^{*}$ \\
\hline & \multicolumn{2}{|c|}{ Positive pregnancy $(n=59)$} & Negative pregnancy ( $n=125)$ & $0.556^{* *}$ \\
\hline & \multicolumn{2}{|l|}{$28(14-44)$} & $26(18-37)$ & \\
\hline \multicolumn{4}{|c|}{$\begin{array}{l}\text { Data are median (interquartile range). } \\
\text { * Jonckheere-Terpestra trend test. } \\
\text { **Wilcoxon rank sum test. } \\
\text { ASA, anti-sperm antibodies }\end{array}$} & \\
\hline
\end{tabular}

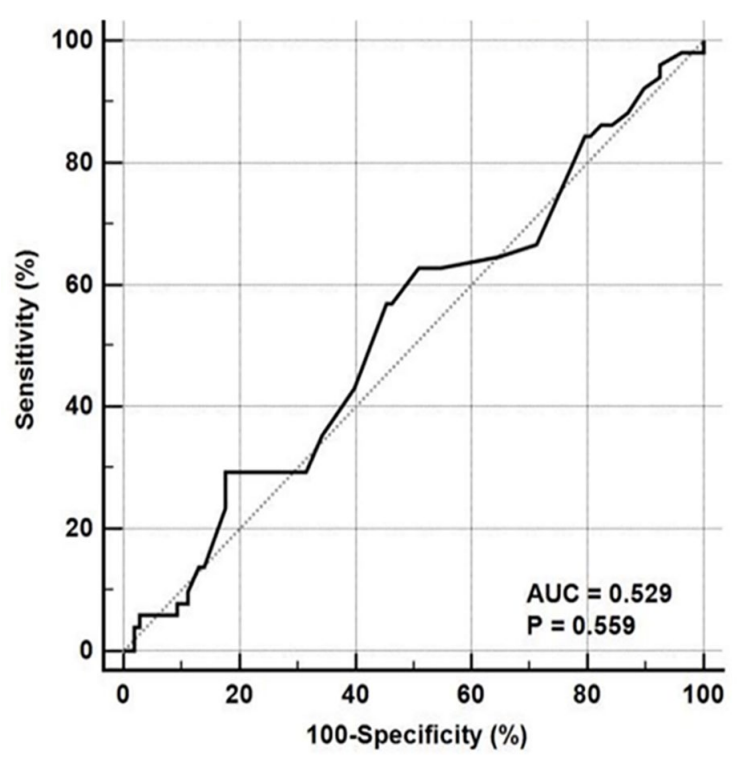

Figure 1. Receiver operating characteristic (ROC) curve analysis for prediction of positive pregnancy using seminal anti-sperm antibodies (ASA) level shows that seminal ASA level had poor predictive value with an area under the ROC curve (AUC) of 0.529 ( $p$-value $=0.559$ )

evaluating the impact of ASA on basic sperm parameters, spontaneous pregnancy rate and ART outcomes leads to more confusion, which may be attributed to the heterogeneity of the studied populations, source of the tested specimens (semen, serum, or cervical secretions), cut-off points, ASA subclasses, methods of testing, and relevance of targeted antigens..$^{21,22}$ Consequently, many clinics have established their own strategies, "normal" ranges, and cut-off points for clinical significance, based on their own point of view regarding the correlation between ASA existence and subsequent treatment outcomes. ${ }^{23}$

As such, we sought to evaluate the influence of seminal plasma ASA on the outcomes of ICSI, in a case-control fashion. ASA could be detected directly on sperm surface using mixed agglutination reaction (MAR) and immunobead test (IBT), which are recommended by the current WHO guidelines due to their wide availability and easy performance, or indirectly in serum or seminal plasma. ${ }^{17,24}$ Unfortunately, no interest was given to the relevance of the targeted antigens, the targeting antibody subclass, the synergetic effect of different ASA subclasses, or the severity of autoimmunization in the current guidelines. ${ }^{25}$ Due to the existence of reliable inaccuracy, inability for quantification of immunoglobulins concentration on sperm surface, high inter-laboratory variability, and inconsistency regarding the recommended cut-off values and interpretation of results in direct MAR and IBT tests for detection of ASA, ${ }^{23}$ and as sample processing and cell fixation used in indirect methods may lead to antigen denaturation and reduction of antigen recognition by ASA, ${ }^{1}$ we preferred to use the ELISA method in the current study for detection of seminal ASA, as a more standardized test, considering the significant correlation between seminal and sperm-bound ASA values. ${ }^{10,26}$ The ELISA test has an additional advantage of the ability to store seminal samples for a long time and run all collected samples in one run, in contrast to IBT and MAR methods which need fresh samples. Interestingly, the ELISA assay, in previous reports, was able to identify antibodies that were negative when screened with the IBT test. ${ }^{27}$ In the present study, the 
prevalence of seminal ASA among our study population was $19 \%$. Data on the frequency of ASA in infertile men have shown high variability among studies (from $5 \%$ to $44 \%$ ), according to the cutoff values and screening method applied. ${ }^{16,19}$ Although sperm count, motility, and morphology were negatively affected by existence of seminal ASA, we did not detect any statistically significant difference between patients with positive and negative seminal ASA regarding fertilization, embryo development, and pregnancy rates after ICSI. Studies conducted by other investigators agree with our results regarding the negative effect of ASA on sperm concentration, ${ }^{28}$ which may be explained by the fact that antibodies stick to the sperm surface, inducing sperm cytotoxicity and apoptosis and enhancing sperm phagocytosis, resulting collectively in a decline in sperm concentration. ${ }^{21}$ Moreover, some researchers reported an inverse relationship between severity of autoimmunization and sperm concentration. ${ }^{29}$

Although some studies supported our results regarding the negative effect of ASA on sperm motility, simply due to slowing down the sperm by joining of antibodies to the sperm tail, 28,30 other investigators did not find any correlation between the existence of ASA and sperm motility, ${ }^{31}$ while a third section of researchers found this negative effect of ASAs on motility to be related to the severity of autoimmunization. ${ }^{29}$ The heterogeneity of the localization of the targeted antigen and its relevance to motility, and the inclusion of cases with unmatched degrees of autoimmunity, may explain the discrepancy between different studies. In contrast to our results, a systematic review conducted in 2015 showed that there was no significant effect of ASA on sperm morphology. ${ }^{21}$ However, it seems difficult to analyze data collected from different studies considering sperm morphology from a statistical point of view, due to the implementation of heterogeneous criteria and cut-off values for morphology evaluation during the past three decades. In our study, although the fertilization rate was lower in patients with positive ASA compared with patients with negative ASA (56.3\% versus $66.7 \%$, respectively), this difference was statistically insignificant $(p=0.091)$. The same results were also reported in other studies. ${ }^{5,16,32}$ Interestingly, a study by Tennakoon and coworkers found that ASA positives have a significantly elevated fertilization rate than ASA negatives $(p$-value $=0.001),{ }^{19}$ which may be explained by the previously reported beneficial effect of some, as yet poorly defined, subclasses of ASA on sperm adhesion to oocyte vestments and the fertilization process. According to biological experimental studies, common epitopes are shared by both sperm and embryos. These epitopes may represent an extra nuclear cleavage signal for the fertilized oocyte and their targeting by specific ASA may impede the process of embryogenesis. ${ }^{33}$ Also, ASA have been suggested to negatively affect successful pregnancy through prevention of implantation of early embryos. ${ }^{12}$ However, in the present study, we found no statistically significant difference between ASApositive and negative groups regarding the embryo quality or the pregnancy outcome, which is supported by the results of similar studies. ${ }^{16,19}$ Also, the current study showed no statistically significant difference between couples with distinct best-produced embryos ( $\mathrm{A}, \mathrm{B}$, or $\mathrm{C}$ ) or pregnancy outcome regarding seminal ASA levels. The proposed negative effect of non-spermbound ASA on the reproductive process may be virtually related to ASA produced by the female genital tract rather than those freely existing in the seminal fluid, which is exposed to filtering processes during semen preparation before ART, removing seminal ASA and preventing any negative impact on ICSI outcomes. Moreover, the undetachable poor-washing-responder spermbound ASA may become inactive or segregated within the ooplasm (like the process that occurs to the acrosome and sperm tail) after microinjection in ICSI, ${ }^{32}$ without apparent subsequent negative consequences, unlike IVF in which these poor-washing-responder sperm-bound antigens may be crucial in key steps necessary for IVF success, such as sperm-zona interaction or oolemma merging, which may explain the inconsistency between both procedures regarding the influence of autoimmunity, ${ }^{13}$ despite subjecting semen samples to the same preparation process. Furthermore, a significantly reduced sperm function was reported by some researchers to be statistically significant only when both $\operatorname{IgA}$ and $\operatorname{IgG}$ antibodies existed, while no significant reduction was reported when either class was present alone. ${ }^{34}$ Other investigators argued that the negative impact on fertilization in IVF programs was more pronounced when $>70 \%$ of spermatozoa were conjugated with dual antibodies subclasses, ${ }^{35}$ which indicates that quantitative as well as qualitative, yet poorly determined, thresholds of ASA, in conjunction with relevant targeted antigens, are prerequisites for the proposed effect of ASA on the reproductive process. 
All the three factors (targeted antigen, dual ASA action, and severity of autoimmunity) are not evaluated thoroughly by the currently available screening tests, representing a major limitation of their diagnostic and prognostic value. Little progress will continue to be made until investigators redirect the aim of research projects from antibody/site-directed testing to antigen-directed testing, to identify specific antigens that, when bound by individual or joined antibody subtypes beyond certain thresholds, would, either negatively or positively, affect fertility.

It may be argued that using the ELISA technique in detection of ASA - in the current study - may led to methodological bias, consistent with overestimation of ASA, due to measurement of some clinically irrelevant seminal ASA directed against sub-surface antigens incorporated with the mix of whole-sperm extract used as a substrate during the procedure. This actually represents a major limitation of the available indirect methods in general, rather than a limitation of a specific study methodology. However, considering the reliable inaccuracy of the WHO-recommended screening tests, we think the ELISA technique may represent the best available alternative, and has the advantage of standardization needed for research purposes, at least till the development of more accurate measurement tool for the detection of clinically relevant ASA.

Since the major limitation of the current study is the relatively small number of ASA-positive patients, we strongly encourage further studies with larger groups of patients, in order to confirm that ICSI outcomes are not impaired by ASA detected by the currently available screening methods.

\section{Conclusion}

Until more specific testing is developed, the routine screening of ASA by the existing screening methods in men prepared for ICSI cannot be recommended for the time being, as ICSI seems to bypass almost all the negative insults created by seminal ASA detected by the currently available methods.

\section{Authors' contributions}

All listed authors (Ahmed F. El-Sherbiny, Tamer A. Ali, Eman A. Hassan, Amira B. Mehaney and Heba A. Elshemy) have performed all four points specified below:
Made substantial contributions to conception and design, acquisition of data, or analysis and interpretation of data; involved in drafting the manuscript or revising it critically for important intellectual content; provided final approval of the version to be published. Each author should have participated sufficiently in the work to take public responsibility for appropriate portions of the content; agreed to be accountable for all aspects of the work in ensuring that questions related to the accuracy or integrity of any part of the work are appropriately investigated and resolved.

All authors read and approved the final manuscript.

\section{Conflict of interest statement}

The authors declare that there is no conflict of interest.

\section{Funding}

The authors received no financial support for the research, authorship, and/or publication of this article.

\section{Ethical approval}

All procedures performed in this study were in accordance with the ethical standards of the Institution and/or National Research Committee and with the 1964 Declaration of Helsinki and its later amendments or comparable ethical standards. The protocol and written informed consent were approved by the local ethical committee of Department of Andrology, International Islamic Center for Population Studies and Research, (affiliated to Al-Azhar University, Egypt) (REC number: 2862). Informed written consent was obtained from all the patients.

\section{ORCID iDs}

\section{Ahmed F. El-Sherbiny (iD) https://orcid.org/0000- 0001-9884-7602}

Tamer A. Ali (iD https://orcid.org/0000-00032640-3425

\section{Availability of data and materials}

The datasets used and/or analyzed during the current study are available from the corresponding author on reasonable request.

\section{References}

1. Vazquez-Levin $\mathrm{MH}$, Marin-Briggiler CI and Veaute C. Antisperm antibodies: invaluable tools toward the identification of sperm proteins 
involved in fertilization. Am $\mathcal{F}$ Reprod Immunol 2014; 72: 206-218.

2. Shai $S$ and Naot Y. Identification of human sperm antigens reacting with antisperm antibodies from sera and genital tract secretions. Fertil Steril 1992; 58: 593-598.

3. Restrepo B and Cardona-Maya W. Antisperm antibodies and fertility association. Actas Urol Esp 2013; 37: 571-578.

4. Lombardo F, Gandini L, Dondero F, et al. Antisperm immunity in natural and assisted reproduction. Hum Reprod Update 2001; 7: 450-456.

5. Lombardo F, Gandini L, Lenzi A, et al. Antisperm immunity in assisted reproduction. f Reprod Immunol 2004; 62: 101-109.

6. Chiu WW and Chamley LW. Clinical associations and mechanisms of action of antisperm antibodies. Fertil Steril 2004; 82: 529-535.

7. Cline AM and Kutteh WH. Is there a role of autoimmunity in implantation failure after in-vitro fertilization? Curr Opin Obstet Gynecol 2009; 21: 291-295.

8. Ford WCL, Williains KM, McLaughlin EA, et al. Immunology: the indirect immunobead test for seminal antisperm antibodies and fertilization rates at in-vitro fertilization. Hum Reprod 1996; 11: 1418-1422.

9. Bates CA. Antisperm antibodies and male subfertility. BfU Int 1997; 80: 691-697.

10. Francavilla F, Santucci R, Barbonetti A, et al. Naturally occurring antisperm antibodies in men: interference with fertility and clinical implications. An update. Front Biosci 2007; 12: 2890-2911.

11. Check JH, Hourani W, Check ML, et al. Effect of treating antibody-coated sperm with chymotrypsin on pregnancy rates following IUI as compared to outcome of IVF/ICSI. Arch Androl 2009; 50: 93-95.

12. Marshburn PB and Kutteh WH. The role of antisperm antibodies in infertility. Fertil Steril 1994; 61: 799-811.

13. Van Weert JM, Repping S, van der Steeg JW, et al. A prediction model for ongoing pregnancy after in vitro fertilization in couples with male subfertility. Reprod Med 2008; 53: 250-256.

14. Vujisic S, Lepej SZ, Jerkovic L, et al. Antisperm antibodies in semen, sera and follicular fluids of infertile patients: relation to reproductive outcome after in vitro fertilization. Am $\mathcal{F}$ Reprod Immunol 2005; 54: 13-20.

15. Baek K, Neri QV, Wang A, et al. Has the advent of ICSI made antisperm antibody testing obsolete? Fertil Steril 2005; 84: S447.

16. Zini A, Fahmy N, Belzile E, et al. Antisperm antibodies are not associated with pregnancy rates after IVF and ICSI: systematic review and metaanalysis. Hum Reprod 2011; 26: 1288-1295.

17. Cooper TG, Noonan E, von Eckardstein S, et al. World Health Organization reference values for human semen characteristics. Hum Reprod Update 2010; 16: 231-245.

18. Baczkowski T, Kurzawa $\mathrm{R}$ and Glabowski W. Methods of embryo scoring in in vitro fertilization. Reprod Biol 2004; 4: 5-22.

19. Tennakoon V, Yasawardene SG and Weerasekera DS. Antisperm antibodies: incidence, isotypes and location on spermatozoa, their implications on fertilization and on pregnancy rate at a selected Centre in Sri Lanka. Sri Lanka J Obstet Gynaecol 2012; 32: 8.

20. Bronson RA, Fusi F, Cooper GW, et al. Antisperm antibodies induce polyspermy by promoting adherence of human sperm to zonafree hamster eggs. Hum Reprod 1990; 5: 690-696.

21. Cui D, Han G, Shang Y, et al. Antisperm antibodies in infertile men and their effect on semen parameters: a systematic review and metaanalysis. Clin Chim Acta 2015; 444: 29-36.

22. Leushuis E, van der Steeg JW, Steures P, et al. Immunoglobulin $\mathrm{G}$ antisperm antibodies and prediction of spontaneous pregnancy. Fertil Steril 2009; 92: 1659-1665.

23. Krapez JA, Hayden CJ, Rutherford AJ, et al. Survey of the diagnosis and management of antisperm antibodies. Hum Reprod 1998; 13: 3363-3367.

24. Shibahara $\mathrm{H}$ and Koriyama J. Methods for direct and indirect antisperm antibody testing. Methods Mol Biol 2013; 927: 51-60.

25. Barbonetti A, Castellini C, D'Andrea S, et al. Relationship between natural and intrauterine insemination-assisted live births and the degree of sperm autoimmunisation. Hum Reprod 2020; 35: 1288-1295.

26. Paul S, Baukloh V and Mettler L. Enzymelinked immunosorbent assays for sperm antibody detection and antigenic analysis. F Immunol Methods 1983; 56: 193-199.

27. Veaute C, Furlong LI, Bronson R, et al. Acrosin antibodies and infertility. I. Detection of 
antibodies towards proacrosin/acrosin in women consulting for infertility and evaluation of their effects upon the sperm protease activities. Fertil Steril 2009; 91: 1245-1255.

28. Verón GL, Molina RI, Tissera AD, et al. Incidence of sperm surface autoantibodies and relationship with routine semen parameters and sperm kinematics. Am $\mathcal{F}$ Reprod Immunol 2016; 76: 59-69.

29. Barbonetti A, Castellini C, D'Andrea S, et al. Prevalence of anti-sperm antibodies and relationship of degree of sperm autoimmunization to semen parameters and postcoital test outcome: a retrospective analysis of over 10000 men. Hum Reprod 20193; 4: 834-841.

30. Rossato M, Galeazzi C, Ferigo M, et al. Antisperm antibodies modify plasma membrane functional integrity and inhibit osmosensitive calcium influx in human sperm. Hum Reprod 2004; 19: 1816-1820.
31. Garcia PC, Rubio EM and Pereira OCM. Antisperm antibodies in infertile men and their correlation with seminal parameters. Reprod Med Biol 2007; 6: 33-38.

32. Esteves SC, Schneider DT and Verza S Jr. Influence of antisperm antibodies in the semen on intracytoplasmic sperm injection outcome. Int Braz F Urol 2007; 33: 795-802.

33. Naz RK. Effects of antisperm antibodies on early cleavage of fertilized ova. Biol Reprod 1992; 46: 130-139.

34. Junk SM, Matson PL, Yovich JM, et al. The fertilization of human oocytes by spermatozoa from men with antispermatozoal antibodies in semen. $\mathcal{F}$ In Vitro Fert Embryo Transf 1986; 3: 350-352.

35. de Almeida M, Gazagne I, Jeulin C, et al. In-vitro processing of sperm with autoantibodies and in-vitro fertilization results. Hum Reprod 1989; 4: 49-53.
Visit SAGE journals online journals.sagepub.com/ home/tau

@SAGE journals 